\title{
Corrigendum
}

\section{Corrigendum to "A Study of Success Rate of Miniscrew Implants as Temporary Anchorage Devices in Singapore"}

\author{
Yi Lin Song, ${ }^{1}$ Mimi Yow, ${ }^{1}$ Ming Tak Chew, \\ Kelvin Weng Chiong Foong, ${ }^{2}$ and Hung Chew Wong ${ }^{3}$ \\ ${ }^{1}$ National Dental Centre Singapore, 5 Second Hospital Avenue, Singapore 168938 \\ ${ }^{2}$ Faculty of Dentistry, National University of Singapore, 11 Lower Kent Ridge Road, Singapore 119083 \\ ${ }^{3}$ Yong Loo Lin School of Medicine, National University of Singapore, 1 E Kent Ridge Road, NUHS Tower Block, \\ Level 11, Singapore 119228 \\ Correspondence should be addressed to Yi Lin Song; song.yi.lin@ndcs.com.sg \\ Received 21 July 2016; Accepted 24 August 2016 \\ Copyright (C) 2016 Yi Lin Song et al. This is an open access article distributed under the Creative Commons Attribution License, \\ which permits unrestricted use, distribution, and reproduction in any medium, provided the original work is properly cited.
}

In the article titled "A Study of Success Rate of Miniscrew Implants as Temporary Anchorage Devices in Singapore" [1], all the authors' first and last names were reversed. The corrected author names are shown above.

\section{References}

[1] S. Y. Lin, Y. Mimi, C. M. Tak, F. K. W. Chiong, and W. H. Chew, "A study of success rate of miniscrew implants as temporary anchorage devices in Singapore," International Journal of Dentistry, vol. 2015, Article ID 294670, 10 pages, 2015. 


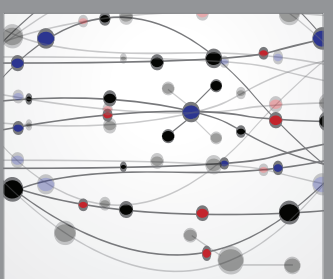

The Scientific World Journal
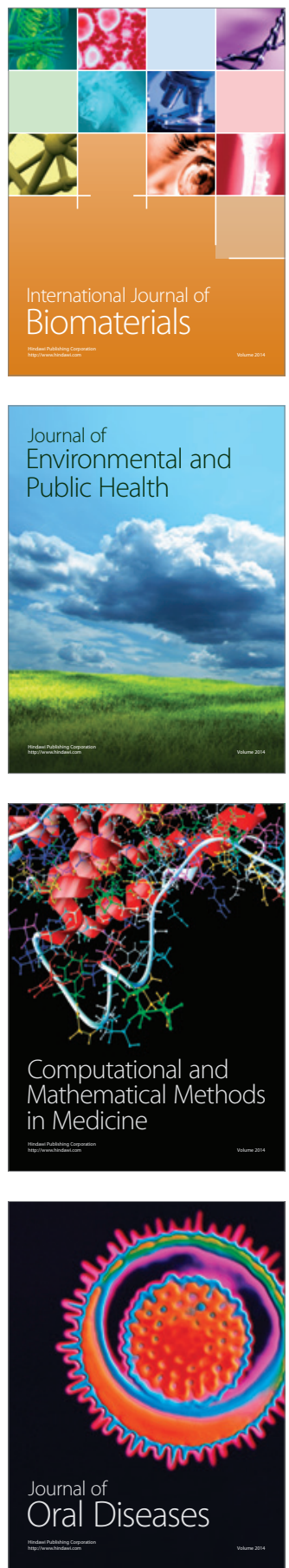
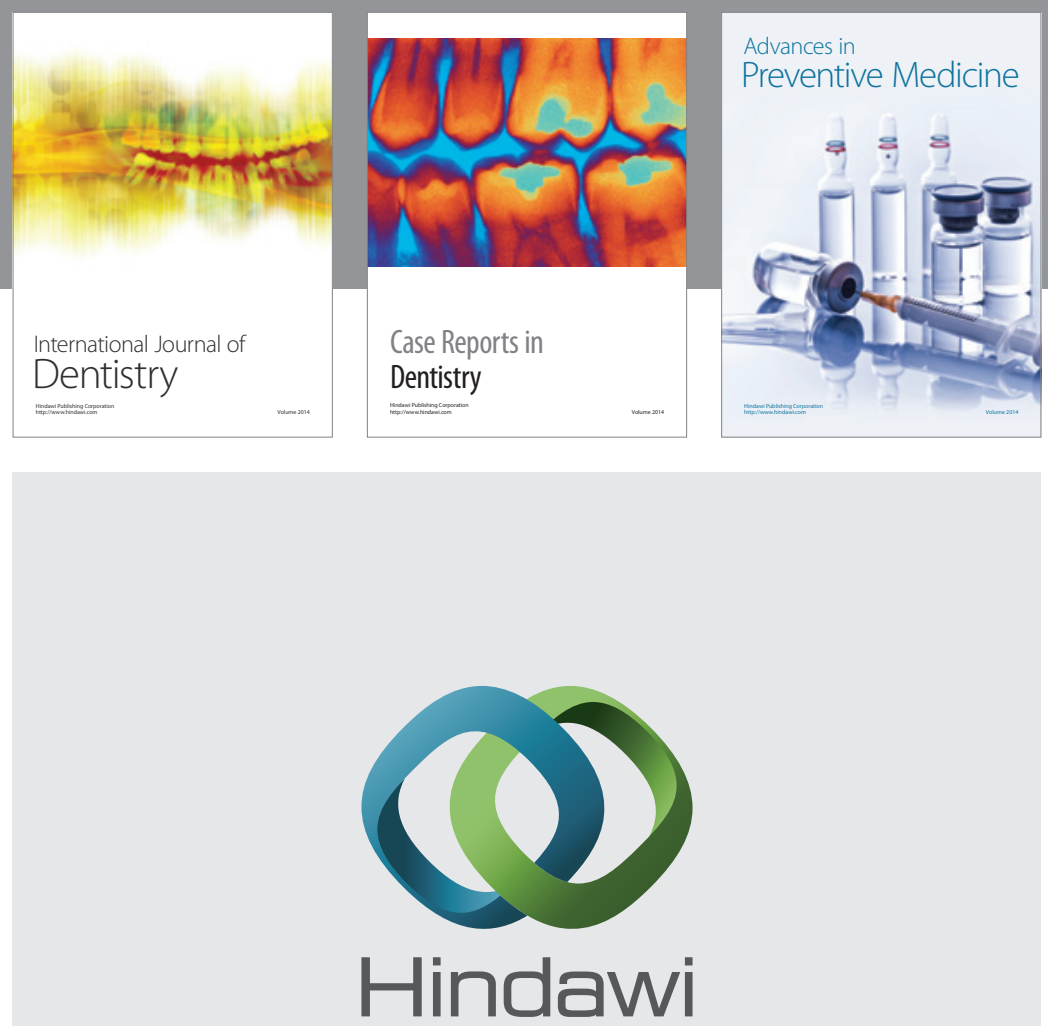

Submit your manuscripts at

http://www.hindawi.com
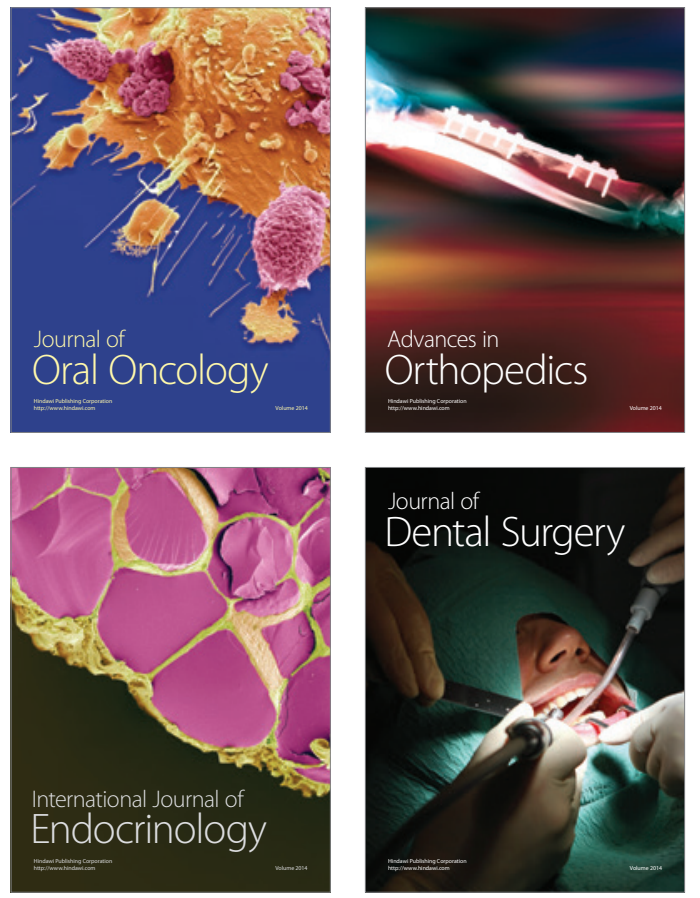
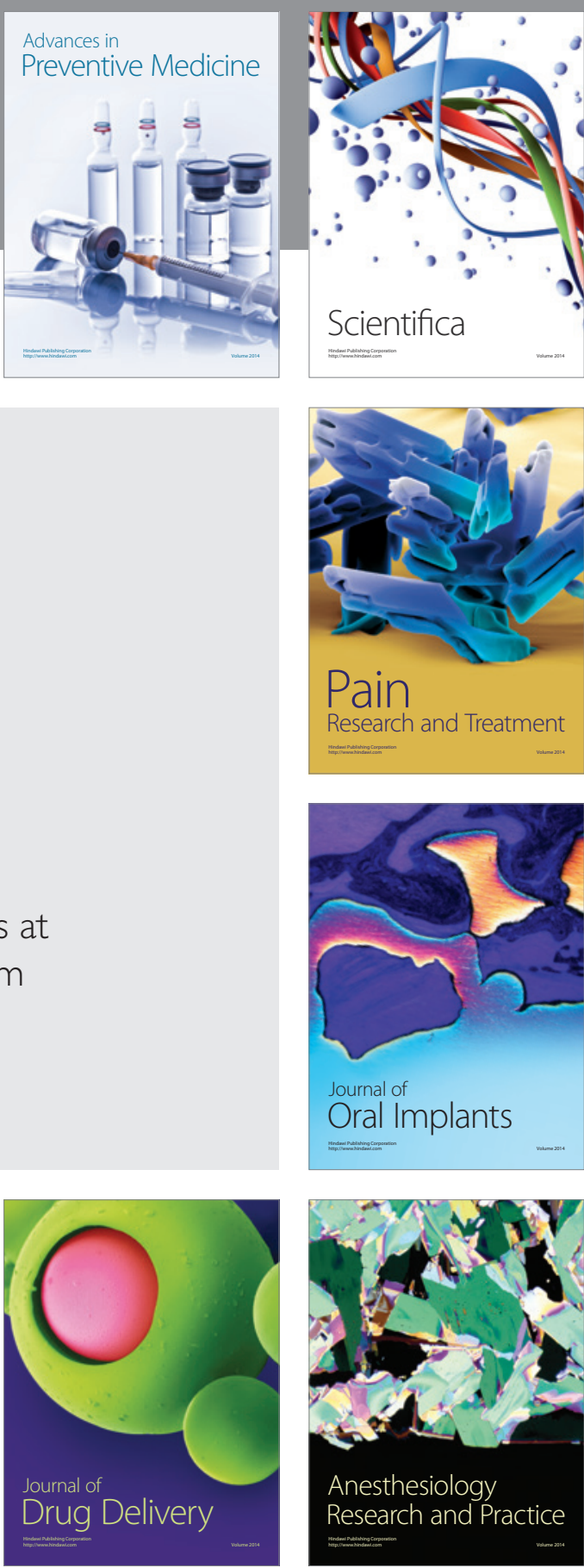

Scientifica
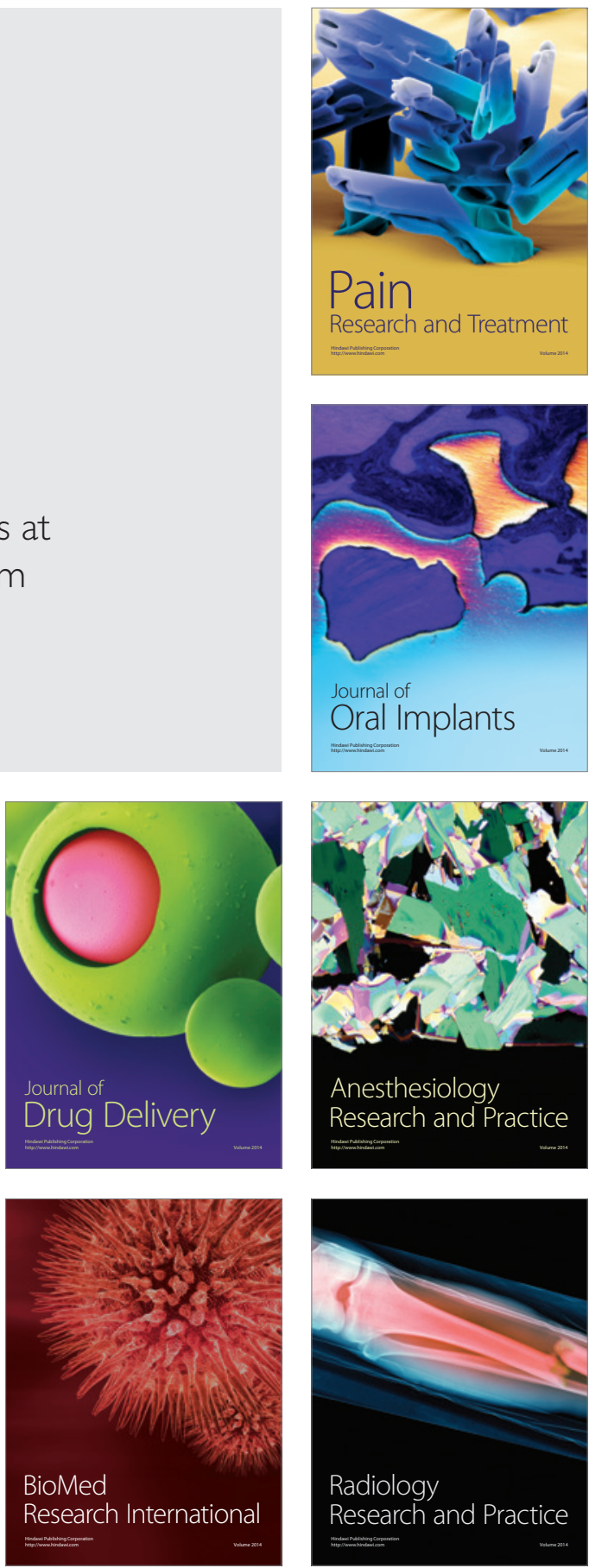